\title{
ON THE PHOTOGRAPHIC VERTICAL CATALOG
}

\author{
V.A.NAUMOV \\ Central Astronomical Observatory, USSR Academy of Sciences \\ Pulkovo \\ 196140 Leningrad \\ USSR
}

The Photographic Vertical Circle (PVC) was constructed in Pulkovo shops in 1962 according to idea of M. Zverev for absolute determinations of declinations of stars. Observations were made at the observatory Serro-Calan in Chile by Chilean and Soviet astronomers in 1964-1966. The PVC is an experimental instrument: it is the first reflector and the first photographic instrument for absolute observations. It has two circles and each circle has four photographic microscopes.

The catalog contains approximately 700 stars from FK4, 700 stars from PFKSZ. Total number of observations is 12762 . The flexure was determined by horizontal collimators. Corrections of divisions of circles were determined by rotation of one circle relatively to the other one. Dependences of observing zenith distances from type of emulsion of photographic plates and colour indexes of stars were found and taken into account. The instrumental errors $\mathrm{O}-\mathrm{C}=0.65 \sin 2 z$ and the jump of O - C in September 1966 were found and taken into account, too.

There was a major problem in calculation of chromatic refraction, since we do not have coefficients of extinction of the Serro-Calan atmosphere. Investigation of corrections for chromatic refraction, using coefficients of extinction for 12 different stations and the theory of Pulkovo astronomers Zhilinsky et al. has shown that differences of this corrections submit to an empiric law , $\Delta z_{\mathrm{ij}}=C_{\mathrm{B}-\mathrm{v}}$ $\tan z$ if $2 \leq m \leq 9, z \leq 75^{\circ}, \mathrm{h} \leq 2000 \mathrm{~m}$, where $i, j$ are indexes of stations, $B-V$ are color indexes of stars, $m$ is magnitude of stars, and $h$ is altitudes of stations.

Therefore it is possible to use coefficients of extinction of some station (i) instead of coefficients of extinction of Serro-Calan atmosphere and to find $C_{\mathrm{i}, \mathrm{j}}$ from observations of lower and upper culminations in Serro-Calan by Bessel's method, if atmospheres of stations $i$ and Serro-Calan submit to this empiric law. Five catalogs were formed by this method. It was found that differences of declinations of these catalogs are less than 0.02 at zenith distance of $45^{\circ}$. The declinations of final version of catalog PVC were formed as arithmetical mean of declinations of these catalogs. It was found that the systematic differences PVC $-\mathrm{FK} 5\left(\Delta \delta_{\delta}, \Delta \delta_{\alpha}, \Delta \delta_{\mathrm{m}}, \Delta \delta_{\mathrm{B}-\mathrm{V}}\right)$ are less than 0.1 .

\section{Discussion}

BASTIAN: You talked about colour-dependent refraction at quite some length. I understood all the details. But I did not get one point: did you discuss this because it is a completely new consideration in your work or because of the special problems at this very observatory?

Naumov: The latter. I discussed it because it could not be done in the usual way. 